\title{
Trainability of leg strength by whole-body electromyostimulation during adult lifespan: a study with male cohorts
}

Institute of Medical Physics, FriedrichAlexander University ErlangenNürnberg, Erlangen, Germany

\section{Simon von Stengel Wolfgang Kemmler}

This article was published in the following Dove Press journal:

Clinical Interventions in Aging

Background: The age-related decline in muscle strength is a well documented phenomenon in human beings. Resistance-type exercise including the novel, joint-friendly, and time-efficient whole-body electromyostimulation (WB-EMS) technology decelerates this unfavorable decline. However, the issue of trainability of the neuromuscular system during different periods of life still remains, especially for WB-EMS. Thus, the aim of this study was to compare the changes in maximum isokinetic leg/hip extensor strength (MIES) and maximum isokinetic leg/hip flexor strength (MIFS) after WB-EMS interventions in men in different periods of life. Our hypothesis was that although WB-EMS significantly increases lower extremity strength in all periods of adults' life, trainability decreases with age with a significantly higher increase at the age of 20-35 years compared with that at the age of $65+$ years.

Subjects and methods: Using an isokinetic leg press, we determined the changes in MIES and MIFS in 118 community-dwelling men aged 27-89 years after 14-16 weeks of WB-EMS interventions applying identical protocols. Men were structured in 15 year-ranged age groups starting at the age of 20-35 years and ending at the age of 80+ years.

Results: Most importantly, WB-EMS-induced gains in MIES and MIFS were significant $(P<0.001)$ in all the groups. Changes in MIFS were on average about twice as high compared with MIES (18-25\% vs 9-15\%). Applying one-way ANOVA, we observed a trend to lower trainability with increasing age $(P=0.060)$ for MIES. Pairwise tests confirmed our hypothesis that the youngest subgroup differs significantly for MIES from men aged $65+$ years $(P=0.007)$. In parallel, one-way ANOVA determined a significant between-group difference $(P=0.046)$ for MIFS; however, we did not determine a significant difference between men aged $<35$ years and $65+$ years.

Conclusion: We observed an inconsistent tendency for blunted WB-EMS-induced lower extremity strength gains in older adults. However, much more importantly, the general effectiveness of WB-EMS to significantly increase maximum hip/leg strength during the adult lifespan can be confirmed.

Keywords: aging, neuromuscular function, muscle strength, lower extremities, trainability, whole-body electromyostimulation, men

\section{Introduction}

Institute of Medical Physics, FriedrichAlexander University Erlangen-Nürnberg, Henkestrasse 91, Erlangen 91052,

Germany

Tel +49 9|3। 8523999

Fax +49 9131 8522824

Email wolfgang.kemmler@imp.unierlangen.de
The age-related decline in muscle strength and function is a well-documented phenomenon in human cohorts. ${ }^{1}$ In excessive cases, this unfavorable trend might result in dyna(mo)penia, ${ }^{1}$ which can be considered as the functional aspect of sarcopenia, ${ }^{2-4}$ a "geriatric syndrome" recently included in the ICD-10, German Modification (ICD-10 GM). In this context, declines in functional performance of the lower 
extremities might be the most crucial physical parameter for frailty and loss of independence in the elderly. ${ }^{6-8}$ Resistance exercise significantly decelerates reductions in muscle strength; ${ }^{1}$ however, the issue of trainability of the neuromuscular system during different periods of life has so far not been consistently answered. ${ }^{12-15}$ Apart from this aspect, the enthusiasm of middle-aged to older people to conduct (intense) exercise protocols adequate to successfully address neuromuscular outcomes ${ }^{16}$ is rather limited. ${ }^{17}$ Whole-body electromyostimulation (WB-EMS), a time-effective, jointfriendly, and highly customizable technology, might thus be a promising candidate for overcoming people's reservations about resistance-type (RT) exercise. In fact, several studies ${ }^{18}$ have reported WB-EMS-induced changes in muscle mass and function in the (lower) range of conventional RT protocols. ${ }^{19,20}$ Nevertheless, the issue of varying trainability of neuromuscular aspects in different periods of life also arises with WB-EMS.

Thus, the general objective of this study was to determine potential differences in the trainability of hip/leg strength indices during adult lifespan when applying similar WB-EMS protocols. To determine functional performance in more depth, ${ }^{21}$ we consistently used a closed kinetic chain testing device in all the studies.

In agreement with studies that observed a blunted response of maximum strength to resistance exercise at higher age, ${ }^{13,14,22}$ our primary hypothesis was that trainability of the maximum dynamic strength of the hip/leg extensors (maximum isokinetic leg/hip extensor strength, MIES) decreases during the adult male lifespan with significant differences between the age of $<35$ and $\geq 65$ years.

Our corresponding core secondary hypothesis was that trainability of the maximum dynamic strength of the hip/leg flexors (maximum isokinetic leg/hip flexor strength, MIFS) decreases during the adult male lifespan with significant differences between the age of $<35$ and $\geq 65$ years.

Another secondary hypothesis (II) was that WB-EMS application resulted in significant improvements with high effect sizes for MIES and MIFS in all the age groups.

Finally, our experimental hypothesis was that the functionally relevant MIFS/MIES index does not change significantly in any of the age groups.

\section{Subjects and methods}

We used the data from previous and ongoing trials, ${ }^{18}$ nonpublished research projects, and university training sessions with nonathletic men of different ages to evaluate the present research issue. The Institute of Medical Physics (IMP),
Erlangen, Germany, planned, conducted, and supervised all the projects or training sessions. In the case of research projects, trials were approved by the university ethics committee of the Friedrich Alexander University (FAU) of ErlangenNürnberg, Germany, and fully complied with the Declaration of Helsinki's "ethical principles for medical research involving human subjects". After detailed information, all participants gave their written informed consent. This also refers to the person as shown in Figure 1 who provided written informed consent for his image to be published.

\section{Outcomes}

- Changes in MIES from baseline to postexercise follow-up.

- Changes in MIFS from baseline to follow-up.

- Changes in MIFS/MIES index from baseline to follow-up.

\section{Participants}

Overall, 118 community-dwelling men aged 27-89 years were included in the study. Inclusion criteria were as follows: 1) male gender, 2) legal age (18+ years), 3) living independently in the community. Subjects were excluded if they were 1) taking medication or suffering from diseases with relevant impact on muscle metabolism; 2) exercising more than two sessions per week; 3) demonstrating low-test compliance or unable to properly perform the isokinetic leg press test; 4 ) attending the corresponding trials with $<80 \%$ of the maximum exercise frequency. Of relevance for the interpretation of the subsequent results, we excluded older institutionalized men and men with diagnosed sarcopenia (exclusion criteria 1) or hip/knee arthrosis (exclusion criteria 3).

\section{Assessments}

Body height was measured by a stadiometer; body mass and composition were determined via direct segmental, multifrequency bio-impedance analysis (DSM-BIA, InBody 230/770; InBody, Seoul, Korea) applying standardized protocols. Baseline characteristics were determined by questionnaires.

MIES/MIFS was tested using an isokinetic leg press (CON-TREX LP; Physiomed, Laipersdorf, Germany; Figure 1). Bilateral hip/leg extension and flexion were performed in a sitting, slightly supine position $\left(15^{\circ}\right)$, supported by hip and chest straps. Range of motion was selected between $30^{\circ}$ and $90^{\circ}$ of the knee angle, with the ankle flexed $90^{\circ}$ and feet firmly fixed with straps positioned on a flexible sliding footplate. The standard default setting of $0.5 \mathrm{~m} / \mathrm{s}$ was used. We consistently used our standard test specifications for 


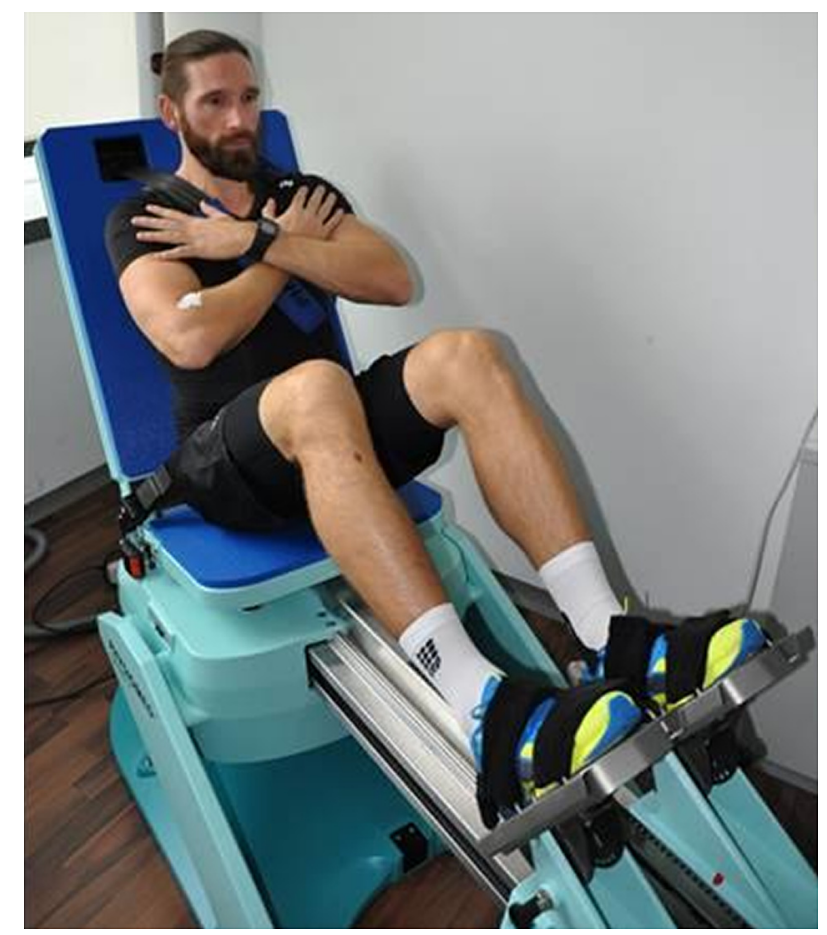

Figure I Hip/leg extensor and flexor strength test conducted on an isokinetic leg press.

all cohorts. Starting with a 5-minute warm up on a cycling ergometer, one familiarization trial (five repetitions) with the dedicated movement pattern (push and pull), and 3 minutes of rest, participants were then asked to conduct five repetitions with maximum voluntary effort. Participants conducted two trials intermitted by 2 minutes of rest. We consistently included the highest value for hip/leg extension and hip/ leg flexion of the five repetitions and both trials in the data analysis. MIFS/MIES index was calculated as MIFS divided by MIES. Reliability for the maximum hip/leg extensor strength (test-retest-reliability; intra-class correlation) was 0.88 (95\% CI: $0.82-0.93$ ) for a male cohort aged $30-50$ years. The same test assessor responsible for the leg press assessments conducted most of the tests.

\section{Intervention}

Longitudinal data were derived from research projects that consistently applied WB-EMS (miha bodytec ${ }^{\circledR}$, Gersthofen, Germany) 1.5 (eg, each Tuesday and every second Thursday) $\times 20$ minutes/week for 14-16 weeks. All the studies used the same WB-EMS protocol with bipolar current, $85 \mathrm{~Hz}, 350 \mu \mathrm{s}$, and duty cycles between 4 and 6 seconds of impulse and 4 seconds of rest. Ten to 14 slight dynamic exercises structured in one to two sets of eight repetitions without any additional weights were performed in a standing position during the 4- to 6-second stimulation phase.
Intensity of the stimulation was regulated in close interaction between the two participants and the instructor using a rate of perceived exertion (RPE) scale to generate a sufficient but tolerable intensity at all the stimulated areas. In all trials, RPE was specified as "6-7" (ie, "hard+" to "very hard") on the Borg CR10 scale. ${ }^{23}$ More details are given in the study by Kemmler et al. ${ }^{24}$

\section{Statistical analyses}

We conducted a post hoc per protocol analysis that only included participants with the eligibility criteria listed earlier. Sample size analysis calculated for the longitudinal part of the study indicated that a group size of 16 participants/ group would generate an $80 \%$ power $(\beta-1)$ to detect a group difference of $5 \pm 5 \%$ (percentage points) with an $\alpha$ of $5 \%$. Of importance, we used pre-post changes in the WB-EMS groups to calculate the percentage changes that were compared between the age groups.

Based on a statistically (Shapiro-Wilk test) and graphically (Q-Q plots) checked normal distribution, the outcomes presented in this study were reported using mean values (MVs) and SD. One-way ANOVA was applied to check group differences for intra-group changes. In the case of relevant differences $(P<0.10)$, pairwise $t$-test comparisons were conducted. Changes within groups were analyzed using dependent $t$-tests. We adjusted for multiple testing of 
our primary hypothesis by applying the Bonferroni-Holm procedure. ${ }^{25}$ Furthermore, we considered lower extremity lean body mass (LBM) changes as a covariable when addressing our primary and core secondary hypotheses. Apart from baseline characteristics (two-tailed significance), we applied single-tailed tests for the primary outcome due to our directed hypotheses. Significance was accepted at $P<0.05$. To adequately address our secondary hypothesis in more depth (II), we also listed effect sizes (ES; Cohen's $\left.d^{\prime}\right)^{26}$ that were based on the net effects (ie, WB-EMS vs control changes) of WB-EMS application on MIES and MIFS in the corresponding trials. The statistical procedures listed earlier were performed using SPSS statistics version 25.

\section{Results}

Table 1 summarizes the baseline characteristics of the 118 men aged 27-89 years included in the present study. Of importance, the number of participants was not equally distributed between the groups (Table 1). Apart from age, groups varied significantly $(P<0.001)$ for body height, total mass and LBM, and physical activity, while differences in body fat rate $(P=0.057)$ were nonsignificant. MVs of anthropometric and lifestyle parameters of the cohort were within the range of the nonathletic German population of comparable age.

Table 2 lists the baseline values and percentage changes in strength indices after 14-16 weeks of WB-EMS application. We observed a trend to lower MIES trainability with increasing age, although one-way ANOVA did not determine any difference between the age groups $(P=0.060)$ for MIES. However, applying a pairwise test, the youngest subgroup differed significantly ( $P=0.007)$ from the cumulated group (ie, 65-79 years and $80+$ years; in detail: $<35$ vs 65-79 years, $P=0.026 ;<35$ vs $80+$ years, $P=0.028$ ) of men 65 years and older. Adjusting on lower extremity LBM changes did not affect this result.

With respect to our core secondary hypothesis, oneway ANOVA determined a significant between-group difference $(P=0.046)$ for MIFS; however, changes for men aged $<35$ years did not significantly differ $(P=0.077)$ from men aged $65+$ years. Adjusting on lower extremity LBM changes did not affect this result.

Considering our secondary hypothesis (II), WB-EMSinduced maximum MIES changes were not only significant $(P<0.001)$ in all the age groups but also demonstrated high effect sizes $\left(d^{\prime} \geq 1.3\right)$. Interestingly, changes in MIFS $\left(P<0.001 ; \mathrm{ES} ; d^{\prime} \geq 1.8\right)$ were on average about twice as high compared with their antagonists (MIES; Table 2).

Due to the much more pronounced changes in MIFS compared with MIES, we determined a significant increase $(P<0.001)$ in the MIFS/MIES index in all the age groups, which did not differ relevantly $(7.9-12.0 \% ; P=0.396)$ between the groups (Table 2), however.

In summary, we confirmed our primary hypothesis that trainability decreases during the adult male lifespan with significant differences between the age of $<35$ and $\geq 65$ years.

Contrary to our expectation, we had to revise our core secondary hypothesis that trainability was significantly higher at the age of $65+$ years compared with that at the age of $<35$ years.

However, also from a pragmatic point of view, the most relevant result, we confirmed our secondary hypothesis II that WBEMS application generates significant improvements in MIES and MIFS that can be considered clinically meaningful.

Finally, we had to revise our experimental hypothesis. In contrast to our, albeit not evidence-based, assumption, the MIFS/MIES index increased significantly in all the

Table I Baseline characteristics of I I8 community-dwelling men aged 27-89 years structured in ranges of I5 years. Data given as mean $\pm S D$

\begin{tabular}{|l|l|l|l|l|l|}
\hline Variables & $<\mathbf{3 5}$ years & $\mathbf{3 5 - 4 9}$ years & $\mathbf{5 0 - 6 4}$ years & $\mathbf{6 5 - 7 9}$ years & $\mathbf{8 0 +}$ years \\
\hline Participants $(\mathrm{n})$ & $\mathrm{I}$ & 31 & $\mathbf{2 4}$ & 32 & 18 \\
\hline Age (years) & $31.1 \pm 3.2$ & $44.2 \pm 4.2$ & $56.6 \pm 4.0$ & $73.8 \pm 4.3$ & $83.5 \pm 2.8$ \\
\hline Body height (cm) & $181.3 \pm 6.3$ & $180.8 \pm 7.4$ & $181.6 \pm 5.3$ & $174.3 \pm 5.9$ & $172.3 \pm 5.6$ \\
\hline Body mass (kg) & $87.2 \pm 11.0$ & $84.7 \pm 10.3$ & $87.0 \pm 10.0$ & $78.8 \pm 8.3$ & $76.0 \pm 10.0$ \\
\hline Lean body mass (kg) & $69.6 \pm 7.8$ & $66.7 \pm 7.8$ & $67.7 \pm 7.0$ & $60.2 \pm 5.5$ & $57.6 \pm 5.5$ \\
\hline Body fat (\%) & $20.2 \pm 6.1$ & $21.3 \pm 7.0$ & $22.3 \pm 5.9$ & $23.6 \pm 4.6$ & $24.2 \pm 4.8$ \\
\hline Activity index & $3.2 \pm 1.2$ & $3.3 \pm 1.3$ & $3.4 \pm 1.5$ & $4.3 \pm 1.3$ & $3.4 \pm 1.5$ \\
\hline Exercise (minutes/week) & $66 \pm 61$ & $61 \pm 55$ & $59 \pm 57$ & $47 \pm 43$ & $38 \pm 33$ \\
\hline Smokers (\%/group) & $12 \%$ & $10 \%$ & $13 \%$ & $9 \%$ & $17 \%$ \\
\hline
\end{tabular}


Table 2 Trainability of MIES and MIFS and the corresponding MIFS/MIES index in 118 community-dwelling men aged 27-89 years structured in groups of I5-year period. Data given as mean \pm SD

\begin{tabular}{|c|c|c|c|c|c|c|}
\hline Variable & $<35$ years & $35-49$ years & $50-64$ years & $65-79$ years & $80+$ years & $P$-value \\
\hline \multicolumn{7}{|c|}{ Maximum isokinetic strength changes (leg press) } \\
\hline Participants (n) & 17 & 31 & 20 & 32 & 18 & - \\
\hline MIES baseline $(\mathrm{N})$ & $3,424 \pm 641$ & $3,304 \pm 520$ & $3,228 \pm 633$ & $2,259 \pm 582$ & $\mathrm{I}, 778 \pm 483$ & 0.001 \\
\hline Changes in MIES (\%) ${ }^{\mathrm{a}}$ & $14.7 \pm 9.9$ & $13.8 \pm 9.8$ & $11.7 \pm 9.2$ & $9.1 \pm 7.3$ & $8.5 \pm 5.5$ & 0.060 \\
\hline MIFS baseline $(\mathrm{N})$ & $1,761 \pm 304$ & $1,520 \pm 317$ & $1,476 \pm 412$ & $970 \pm 234$ & $748 \pm 196$ & 0.001 \\
\hline Changes in MIFS (\%) ${ }^{\mathrm{a}}$ & $23.2 \pm 10.7$ & $24.3 \pm 9.3$ & $24.7 \pm 11.7$ & $\mid 8.1 \pm 11.0$ & $17.9 \pm 9.5$ & 0.046 \\
\hline Index baseline & $0.52 I \pm 0.109$ & $0.463 \pm 0.084$ & $0.453 \pm 0.092$ & $0.429 \pm 0.073$ & $0.42 I \pm 0.54$ & 0.002 \\
\hline Change index (\%) & $7.9 \pm 8.4$ & $9.7 \pm 9.5$ & $12.0 \pm 9.5$ & $8.6 \pm 11.6$ & $8.8 \pm 8.8$ & 0.396 \\
\hline
\end{tabular}

Note: aAll intra-group (ie, pre-post) changes were $P \leq 0.001$.

Abbreviations: MIES, maximum isokinetic leg/hip extensor strength; MIFS, maximum isokinetic leg/hip flexor strength.

age groups without any significant differences between the groups.

\section{Discussion}

Within the WB-EMS framework, we determined the agedependent trainability of lower extremity performance using a functionally meaningful closed kinetic chain test (isokinetic leg press). ${ }^{21}$ In summary, WB-EMS significantly increased maximum lower extremity strength in all the age groups ranging from $<35$ years to $80+$ years. However, we were unable to clearly decide whether trainability of maximum lower extremity strength varies between different periods of adult lifespan or not. Although we observed a tendency of lower trainability in higher age, a significant difference between men aged $<35$ years compared with men aged 65 years and older was observed for MIES but not for MIFS. Due to changes in maximum dynamic hip/leg flexor strength about twice as high compared with extensor strength, the MIFS/MIES index improved significantly in all the age groups without significant intergroup differences. It is difficult to conclude the relevance of this parameter, as it did not perfectly reflect the hamstring-quadriceps $(\mathrm{H}-\mathrm{Q})$ index as a recognized marker of strength balance of muscles spanning the knee joint in the field of fitness and rehabilitation. ${ }^{27,28}$ However, considering the significant and rather linear decline in the MIFS/MIES index during adult lifespan (Table 2), we speculate that this development might contribute to a more functionally lower extremity strength balance. ${ }^{29}$

Due to the lack of corresponding WB-EMS approaches, only a few trials ${ }^{12-15,30,31}$ properly compared trainability of lower extremity muscle strength between different periods of life. Early research ${ }^{22}$ reported a very pronounced decline in trainability. Quantifying this reduction, trainability of men aged 65 years averaged only one-third of the maximum trainability $(=100 \%)$ at the age of 25 years. The corresponding decline in our oldest group (80+ years) was considerably lower (MIES: 42\%; MIFS: 23-27\%); furthermore, all groups significantly gained MIES and MIFS. Comparing young ${ }^{14,15,31}$ or middle-aged ${ }^{12,13,15,31}$ vs old men, two studies ${ }^{13,14}$ reported significantly higher lower extremity strength gains in younger/ middle-aged cohorts after 24-26 weeks of RT $(\approx 70-75$ or $\approx 85 \%$ one repetition maximum $[1 \mathrm{RM}]$ ). On the other hand, three studies ${ }^{12,15}$ detected no, or only nonsignificant, trends for blunted maximum lower extremity strength or power ${ }^{32}$ gains at higher age after 8,12 , and 20 weeks of moderateto-high intensity RT ( $70 \%-80 \% 1 \mathrm{RM})$. One study ${ }^{31}$ reported heterogeneous data for their two lower leg muscle groups assessed. Of importance, results were independent of study length, ${ }^{13,15}$ relative exercise intensity, ${ }^{12-15,31,32}$ or whether open or closed kinetic chain exercise tests ${ }^{14}$ were applied. This is of relevance, as all of these parameters had been considered as potential confounders of age-dependent trainability. Due to the pronounced recruitment and increase in type II fibers ${ }^{33}$ and the lower degree of functionality, WB-EMS might not perfectly represent RT; however, in summary, our result confirmed the slight tendency of blunted trainability of maximum lower extremity strength during aging in men.

Apart from the retrospective analysis of different WBEMS trials, the study featured some particularities and limitations that might decrease the evidence of our results or at least affect the proper transformation to RT. 1) The groups were not equally "manned" with a relative lack of participants between the age group of 61 and 69 years $(n=9)$. Furthermore, although higher than in most other corresponding studies, ${ }^{12-15,34-36}$ the small sample size in some groups (eg, <35 years: $\mathrm{n}=17$ ) might slightly impair our results. 
2) We focused on community-dwelling men without relevant orthopedic conditions (eg, sarcopenia and knee/hip arthrosis). Although this approach enables a proper addressing of our hypothesis, it is debatable whether our corresponding group represented the cohort of community dwelling men aged 80 years and older. However, although it may sound plausible that people with sarcopenia demonstrate a lower trainability of maximum strength than their healthy peers, when adjusting age-dependent changes in MIES and MIFS for lower extremity LBM, we did not observe any difference to the non-adjusted result. 3) We included solely male cohorts in this comparison of age-related difference of lower extremity trainability. Thus, the transformation of our results to female cohorts might be limited, although the majority of studies reported no corresponding gender differences. ${ }^{13,35,37,38}$ 4) We focused solely on MIES and MIFS as assessed by a functionally meaningful closed kinetic chain systems. Although we were aware that upper extremity muscle strength (eg, handgrip) is also very important for older people ${ }^{9-11}$ due to its crucial role for mobility, independency, frailty, and mortality of older people; ${ }^{6-8,39}$ lower extremity strength might be the more relevant functional parameter. This viewpoint was supported by a longitudinal study 40 that reported a much higher association of knee extension strength and mortality in older people compared with hand grip strength. 5) One may argue that a comparison of the net effect (WB-EMS vs control) might be more meaningful than our approach of focusing on pre-post changes in the WB-EMS groups only. However, apart from reduced statistical power due to a smaller sample sizes in some of the control groups (eg, 80+ years), the rather short study period (14-16 weeks) of the trials resulted in negligible changes in MIES and MIFS in the control groups (ES: $d^{\prime}<0.1 ; P>0.0 .2$ ). Correspondingly, within-group changes in the WB-EMS trials widely overlap with differences between WB-EMS and control. 6) We opted against a more sophisticated statistical analysis, eg, a change point analysis of linear trends, due to the limitations listed earlier. Furthermore, we adjusted the statistical comparison of MIES and MIFS changes in men aged $<35$ vs $\geq 65$ years only for changes in lower extremity LBM. One may argue that medication and/or clinical conditions of increased age might also confound our result and should therefore be statistically considered. However, taking into account that men on medication (eg, glucocorticoids) or with diseases (eg, sarcopenia) with a relevant effect on muscle metabolism and/or conditions (eg, knee/hip arthrosis) so as to prevent proper leg press performance were excluded, we thought that there is no need to adjust for less relevant, subordinate conditions (eg, hypertonia). In summary, however, we thought that our statistical approach adequately addressed our research questions. 7) Although there is a close correlation with narrow limits of agreement between the DSM-BIA InBody 230 and InBody 770 (Seoul, Korea), each applied in the dedicated trials of this project, we also abstained from discussing these anthropometric data in more detail. 8) Although the WB-EMS protocol per se was highly standardized, instructors, crucial for the regulation of the strain intensity, varied between the groups. 9) Only two WBEMS electrodes each (ie, thigh and gluteus) focus on muscles relevant for MIES/MIFS. 10) Due to recruitment of the large hip extensor muscles, the MIFS/MIES index determined by closed kinetic chain testing may vary considerably from the index of 0.6 reported as a normative value for the $\mathrm{H}-\mathrm{Q}$ index when applying open kinetic chain testing. ${ }^{29}$

\section{Conclusion}

In summary, we observed at least a tendency for blunted WBEMS-induced lower extremity strength gains in older adults. However, considering that maximum lower leg strength is a crucial parameter for independent living, the evidence that WB-EMS significantly increases in all the age groups might be the most striking result of this comparison. Furthermore, for the lack of corresponding trials that reported the rate of leg flexion vs leg extension strength ( $\mathrm{H}-\mathrm{Q}$ index) in nonathletic populations applying closed kinetic chain systems, we considered the significant increase in MIFS/MIES index as a beneficial result.

\section{Acknowledgment}

This study was exclusively funded by the resources of the Institute of Medical Physics, Friedrich-Alexander University Erlangen-Nürnberg.

\section{Author contributions}

Both authors contributed toward data analysis, drafting and critically revising the paper, gave final approval of the version to be published, and agree to be accountable for all aspects of the work.

\section{Disclosure}

The authors report no conflicts of interest in this work.

\section{References}

1. Mitchell WK, Williams J, Atherton P, Larvin M, Lund J, Narici M. Sarcopenia, dynapenia, and the impact of advancing age on human skeletal muscle size and strength; a quantitative review. Front Physiol. 2012;3:260. 
2. Cruz-Jentoft AJ, Baeyens JP, Bauer JM, et al. Sarcopenia: European consensus on definition and diagnosis: Report of the European Working Group on Sarcopenia in Older People. Age Ageing. 2010;39(4):412-423.

3. Fielding RA, Vellas B, Evans WJ, et al. Sarcopenia: an undiagnosed condition in older adults. Current consensus definition: prevalence, etiology, and consequences. International working group on sarcopenia. J Am Med Dir Assoc. 2011;12(4):249-256.

4. Studenski SA, Peters KW, Alley DE, et al. The FNIH sarcopenia project: rationale, study description, conference recommendations, and final estimates. J Gerontol A Biol Sci Med Sci. 2014;69(5):547-558.

5. Cruz-Jentoft AJ, Landi F, Topinková E, Michel JP. Understanding sarcopenia as a geriatric syndrome. Curr Opin Clin Nutr Metab Care. 2010;13(1):1-7.

6. Caballero B, Rubio-González A, Potes Y, et al. Associations of the antioxidant capacity and hemoglobin levels with functional physical performance of the upper and lower body limbs. Age. 2014;36(2):851-867.

7. Coto Montes A, Boga JA, Bermejo Millo C, et al. Potential early biomarkers of sarcopenia among independent older adults. Maturitas. 2017;104:117-122.

8. Roshanravan B, Patel KV, Fried LF, et al. Association of Muscle Endurance, Fatigability, and Strength With Functional Limitation and Mortality in the Health Aging and Body Composition Study. J Gerontol A Biol Sci Med Sci. 2017;72(2):284-291.

9. Izawa KP, Watanabe S, Osada N, et al. Handgrip strength as a predictor of prognosis in Japanese patients with congestive heart failure. Eur J Cardiovasc Prev Rehabil. 2009;16(1):21-27.

10. Leong DP, Teo KK, Rangarajan S, et al. Prognostic value of grip strength: findings from the Prospective Urban Rural Epidemiology (PURE) study. Lancet. 2015;386(9990):266-273.

11. Sayer AA, Kirkwood TB. Grip strength and mortality: a biomarker of ageing? Lancet. 2015;386(9990):226-227.

12. Häkkinen K, Pakarinen A. Serum hormones and strength development during strength training in middle-aged and elderly males and females. Acta Physiol Scand. 1994;150(2):211-219.

13. Häkkinen K, Pakarinen A, Kraemer WJ, Newton RU, Alen M. Basal concentrations and acute responses of serum hormones and strength development during heavy resistance training in middle-aged and elderly men and women. J Gerontol A Biol Sci Med Sci. 2000;55(2) B95-B105.

14. Lemmer JT, Martel GF, Hurlbut DE, Hurley BF. Age and sex differentially affect regional changes in one repetition maximum strength. J Strength Cond Res. 2007;21(3):731-737.

15. Phillips BE, Williams JP, Greenhaff PL, Smith K, Atherton PJ. Physiological adaptations to resistance exercise as a function of age. JCI Insight. 2017;2(17).

16. American College of Sports Medicine, Chodzko-Zajko WJ, Proctor DN, et al. American College of Sports Medicine position stand. Exercise and physical activity for older adults. Med Sci Sports Exerc. 2009;41(7): 1510-1530.

17. Carlson SA, Fulton JE, Schoenborn CA, Loustalot F. Trend and prevalence estimates based on the 2008 Physical Activity Guidelines for Americans. Am J Prev Med. 2010;39(4):305-313.

18. Kemmler W, Weissenfels A, Willert S, et al. Efficacy and safety of low frequency whole-body electromyostimulation (WB-EMS) to improve health-related outcomes in non-athletic adults. A systematic review. Front Physiol. 2018;9:573.

19. Peterson MD, Rhea MR, Sen A, Gordon PM. Resistance exercise for muscular strength in older adults: a meta-analysis. Ageing Res Rev. 2010;9(3):226-237.

20. Peterson MD, Sen A, Gordon PM. Influence of resistance exercise on lean body mass in aging adults: a meta-analysis. Med Sci Sports Exerc. 2011;43(2):249-258
21. Augustsson J, Thomeé R. Ability of closed and open kinetic chain tests of muscular strength to assess functional performance. Scand J Med Sci Sports. 2000;10(3):164-168.

22. Hettinger T. Die Trainierbarkeit menschlicher Muskeln in Abhäingigkeit vom Alter und Geschlecht. Int Z angew Physiol einschl Arbeitsphysiol. 1958; 17:371-377.

23. Borg E, Kaijser L. A comparison between three rating scales for perceived exertion and two different work tests. Scand J Med Sci Sports. 2006;16(1):57-69.

24. Kemmler W, Teschler M, Weißenfels A, et al. Effects of Whole-Body Electromyostimulation versus High-Intensity Resistance Exercise on Body Composition and Strength: A Randomized Controlled Study. Evid Based Complement Alternat Med. 2016;2016:1-9.

25. Holm S. A Simple Sequentially Rejective Multiple Test Procedure. Scand J Stat. 1979;6(2):65-70.

26. Cohen J. Statistical Power Analysis for the Behavioral Sciences. 2nd ed. Hillsdale, NJ: Lawrence Erlbaum Associate; 1988.

27. Aagaard P, Simonsen EB, Trolle M, Bangsbo J, Klausen K. Isokinetic hamstring/quadriceps strength ratio: influence from joint angular velocity, gravity correction and contraction mode. Acta Physiol Scand. $1995 ; 154(4): 421-427$.

28. Hiemstra LA, Webber S, Macdonald PB, Kriellaars DJ. Hamstring and quadriceps strength balance in normal and hamstring anterior cruciate ligament-reconstructed subjects. Clin J Sport Med. 2004;14(5) 274-280.

29. Coombs R, Garbutt G. Developments in the use of the hamstring/ quadriceps ratio for the assessment of muscle balance. J Sports Sci Med. 2002;1(3):56-62.

30. Izquierdo M, Häkkinen K, Antón A, et al. Maximal strength and power, endurance performance, and serum hormones in middle-aged and elderly men. Med Sci Sports Exerc. 2001;33(9):1577-1587.

31. Psatha M, Wu Z, Gammie F, et al. Age-related changes in the effects of strength training on lower leg muscles in healthy individuals measured using MRI. BMJ Open Sport Exerc Med. 2017;3(1):e000249.

32. Jozsi AC, Campbell WW, Joseph L, Davey SL, Evans WJ. Changes in power with resistance training in older and younger men and women. J Gerontol A Biol Sci Med Sci. 1999;54(11):M591-M596.

33. Kern H, Barberi L, Löfler S, et al. Electrical stimulation counteracts muscle decline in seniors. Front Aging Neurosci. 2014;6:189.

34. Häkkinen K, Alen M, Kallinen M, Newton RU, Kraemer WJ. Neuromuscular adaptation during prolonged strength training, detraining and re-strength-training in middle-aged and elderly people. Eur J Appl Physiol. 2000;83(1):51-62.

35. Häkkinen K, Kallinen M, Linnamo V, Pastinen UM, Newton RU, Kraemer WJ. Neuromuscular adaptations during bilateral versus unilateral strength training in middle-aged and elderly men and women. Acta Physiol Scand. 1996;158(1):77-88.

36. Izquierdo M, Häkkinen $\mathrm{K}$, Ibañez J, et al. Effects of strength training on muscle power and serum hormones in middle-aged and older men. J Appl Physiol. 2001;90(4):1497-1507.

37. Fiatarone MA. High-Intensity Strength Training in Nonagenarians. JAMA. 1990;263(22):3029-3034.

38. Pyka G, Taaffe DR, Marcus R. Effect of a sustained program of resistance training on the acute growth hormone response to resistance exercise in older adults. Horm Metab Res. 1994;26(7):330-333.

39. Visser M, Goodpaster BH, Kritchevsky SB, et al. Muscle mass, muscle strength, and muscle fat infiltration as predictors of incident mobility limitations in well-functioning older persons. J Gerontol A Biol Sci Med Sci. 2005;60(3):324-333.

40. Laukkanen P, Heikkinen E, Kauppinen M. Muscle strength and mobility as predictors of survival in 75-84-year-old people. Age Ageing 1995;24(6):468-473. 


\section{Publish your work in this journal}

Clinical Interventions in Aging is an international, peer-reviewed journal focusing on evidence-based reports on the value or lack thereof of treatments intended to prevent or delay the onset of maladaptive correlates of aging in human beings. This journal is indexed on PubMed Central, MedLine,

CAS, Scopus and the Elsevier Bibliographic databases. The manuscript management system is completely online and includes a very quick and fair peer-review system, which is all easy to use. Visit http://www.dovepress. com/testimonials.php to read real quotes from published authors. 